\title{
Characterization of Indoor Small Cells Propagation
}

\author{
Rooderson M. Andrade, Rui R. Paulo, Salomão M. Francisco, Emanuel Teixeira and Fernando J. Velez \\ Instituto de Telecomunicações and Universidade da Beira Interior \\ Faculdade de Engenharia, Departamento de Engenharia Electromecânica \\ Covilhã, Portugal \\ 0000-0001-6070-1595, 0000-0002-8541-6675, 0000-0002-5431-3163, 0000-0002-6546-5277, 0000-0001-9680-123X
}

\begin{abstract}
The characterization of the wireless medium in indoor small cell networks is essential to obtain appropriate modeling of the propagation environment. Universal Software Radio Peripherals (USRPs) and simple dipole antennas can emulate LTE-Advanced networks. In this work, we verify WINNER II propagation modeling for the indoor femtocell environment by considering different classrooms of $7.32 \times 7.32$ square meters near a common University Department corridor while measuring the power received in UEs placed in a grid of 49 points (radiated by the small eNodeB in the centre of the classroom of the own cell). These measurements have been carried out either by using the Software Radio Systems LTE that emulates the LTEAdvanced network and its UEs, or by measuring the received power in the UES with a Rohde \& Schwarz FSH8 spectrum analyzer. In room 1, by varying the $U E$ position, the highest values of the received power have occurred close to the central BS, and then in the opposite wall, further away from the interferer. Nevertheless, it was verified that the received power does not decrease suddenly because of the effect of the radiation pattern of the BS and UE antennas for large angles of apertures, as well as due to the non-omnidirectional horizontal antenna pattern. In addition, it was demonstrated that there is an effect of "wall loss" proven by the fact that path loss increases between room 2 and room 1 (or between room 3 and 2). If we consider an attenuation for each wall of circa 7-9 dB the behavior of the WINNER II model at $2.625 \mathrm{GHz}$ for the interference coming across different walls is verified.
\end{abstract}

Index Terms-Propagation, OFDM, small cell networks, LTEAdvanced, srsLTE, WINNER II model.

\section{INTRODUCTION}

To answer to the increasing user demand, mobile networks toward 5G has evolving heterogeneous networks, with differentiation of Evolved NodeBs (eNBs) coverage, as established by 3GPP Release 16 [1]. Small cells, overlaid by larger cells, can be deployed either indoor or outdoor, to provide indoor coverage, outdoor coverage, or even to provide indoor coverage from transmitter nodes placed outdoor.

It is important to explore strategies to increase LTEAdvanced (LTE-A) end-to-end service reliability of mobile multimedia exchanges, e.g., video streaming. One option is to use Universal Software Radio Peripherals (USRPs) that emulate 4G network [2], [3] in indoor or outdoor deployment scenarios, in conjunction with Software Radio Systems LTE

This work is funded by Marie Skłodowska-Curie Actions (MSCA) ITN TeamUp5G (813391), ORCIP, and by UIDB/50008/2020. TeamUp5G project has received funding from the European Union's Horizon 2020 research and innovation programme under the Marie Skłodowska-Curie project number 813391.
(srsLTE), an open source library for the PHY layer of LTEA written in ANSI C. It includes a complete software radio [4] and provides interfaces for the Universal Hardware Driver (UHD) that supports USRPs [5].

Research on the characterization of these femtocell environments at $2.6,3.5$ or $5.62 \mathrm{GHz}$ is still of great interest. Authors from [6], [7], have made their experiments in linearshaped paths within indoor scenarios where the user equipment (UE) and transmitter $(T X)$ eNodeBs are few meters apart. The path loss is affected not only by factors such as architectural structures, including the material and the thickness of the walls, diffraction, reflection and interference [8] but also by the distance between the transmitter and receiver $(T x)$ and receiver $(R x)$.

The objective of this paper is to investigate the coverage and co-channel interference trade-off in OFDM-based indoor small cells with a given reuse pattern $(k)$. A small cell LTEA University environment is considered. Although the LTE-A downlink (DL) is assumed, results can easily be generalized to 5G New Radio cellular networks. First, we consider an analytical approach to compute the signal-to-interference plus noise ratio (SINR) by considering the WINNER II propagation model [9]. Second, an experimental field is performed test in an indoor scenario using srsLTE with a set of USRPs B210 to measure the quality of the received signal as a function of the distance, either by determining the received power $\left(P_{r}\right)$ or computing the carrier-to-interference ratio, $C / I$, either with the USRP B210 used as a UE (and measurement device) or by using a Rohde \& Schwarz FSH8 spectrum analyzer. UE receives data packets from the own cell (eNodeB of interest), while the other eNodeBs only cause interference.

In our experiments, we have measured the received power in different class rooms from our Department while verifying the Winner II propagation model. The USRP B210 has a highspeed serial interface for integration with other boards, allowing the improvement of SISO systems [10]. Its transmitter power is in between 50 and $200 \mathrm{~mW}$. A temporary license was assigned to us by ICP-ANACOM, the Portuguese regulator, to perform field tests in the $2625 \mathrm{MHz}$ (DL) and 2505 (uplink) $\mathrm{MHz}$ frequency bands, each with bandwidth of $10 \mathrm{MHz}$.

The rest of the paper is organized as follows. In Sec. II we present a theoretical study on the average SINR. Sec. III presents the experimental setup with the USRPs that emulate $4 \mathrm{G}$ networks as well results for the $C / I$ (not SINR, as the wireless system is interference-limited) obtained the USRPs 
as measurement devices. Conclusions are finally drawn in Sec. IV, followed by suggestions for future research.

\section{AVERAGE SignAL-TO-INTERFERENCE-PLUS-NOISE RATIO}

\section{A. Scenario}

The deployment scenario presented in Fig. 1 is considered. The geometry corresponds to a real scenario, in a floor with

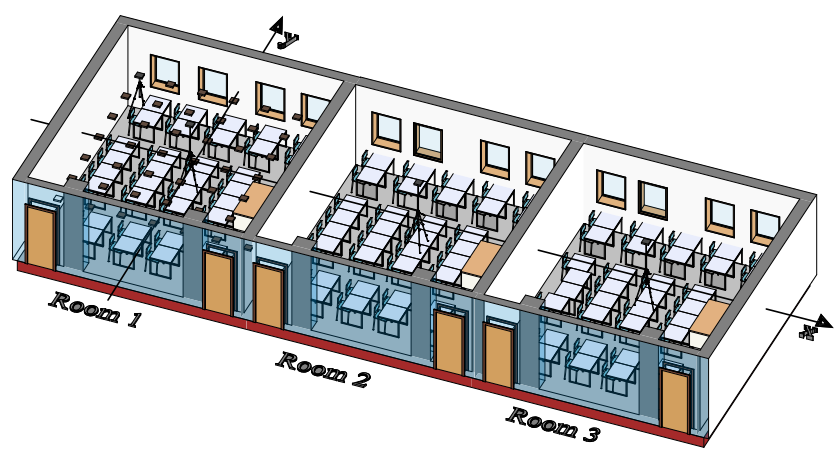

Fig. 1: Characterization of the classrooms in the indoor testing scenario.

three adjacent class rooms placed next to the Instituto de Telecomunicações Laboratory of the Department of Electromechanical Engineering at the University da Beira Interior. The dimensions of each room are $7.32 \times 7.32 \mathrm{~m}^{2}$. Room 1 is the classroom of interest, where received power/interference measurements took place. In this experiments, in room 1 and room 2 the whiteboards have been removed, while in room 3 the whiteboard has been kept. Wireless nodes at rooms 2 or 3 act as interfering BSs.

Measurements have been performed at the points from the grid presented in Fig. 2, by collecting values measured by the USRP (or the spectrum analyzer), in each of the forty-nine points, at room 1 .

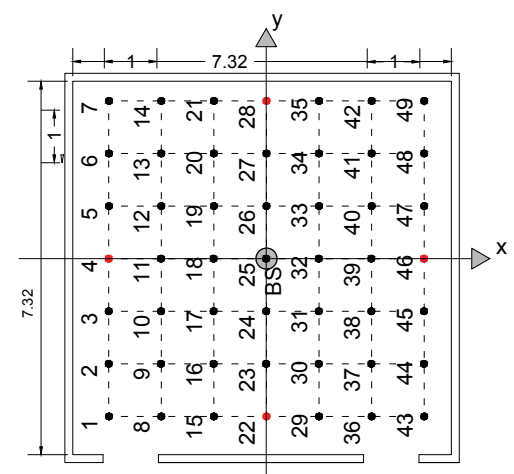

Fig. 2: Distribution of UEs in the measurements.

During the field tests all the furniture was kept inside the classrooms but the white boards were removed form the walls. Descriptive statistics, was considered to calculate the dispersion of the measurement results in relation to the means, whilst considering the normality test and the underlying standard deviation (SD), confidence interval (CI), maximum (Max) and minimum (Min) values for the received power. The $C / I$ has also been calculated.

\section{B. Theoretical Average SINR}

The first step before the experiments consists of making a theoretical study of the average SINR within the considered deployments. Let us consider as the first scenario only the nodes in the coordinates $(0,0)$ and $(0, l)$, as presented in Fig. 3. Each mode serves the room where it was deployed.

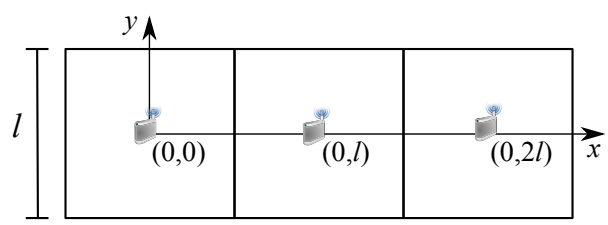

Fig. 3: Testing scenario: the node of interest is in room 1 while the interferer node is either in room 2 or in room 3.

Since frequency reuse pattern one is considered each node adds interference to the served area of the active neighbor node. In this first topology, at room 3 , in the coordinate $(0$, $2 \times l$ ) there is not any node. It is assumed that the node in the room only serves users in that room at any point with coordinate $(x, y)$, whose boundary is delimited by the walls of room 1 , served by the node in the coordinate $(0,0)$. The SINR is calculated through an approach similar to the one described in [11], [12], as follows:

$$
\operatorname{SINR}\left(P_{T x}, x, y\right)=\frac{P\left(P_{T x}, 0,0\right)}{P\left(P_{T x}, 0, l\right)+P_{\text {noise }}},
$$

where $P\left(P_{T x}, 0,0\right)$ is the received power from the own cell, served by the node in $(0,0) . P\left(P_{T x}, 0, l\right)$ is the interference power from the neighbor cell in $(0, l) . P_{\text {noise }}$ is the thermal noise power, in $\mathrm{dBW}$, and is defined by:

$$
P_{\text {noise }}=-174+10 * \log _{10} B W-30+N F,
$$

where the bandwidth is $B W=10 \mathrm{MHz}$ and the noise figure, $N F$, is considered to be $8 \mathrm{~dB}$ (in LTE, $N F$ is typically 7-9 $\mathrm{dB}$ [13]).

In the considered scenario, $P\left(P_{T x}, 0,0\right)$ and $P\left(P_{T x}, 0, l\right)$ are computed as follows:

$$
P\left(P_{T x}\right)=P_{T x} G_{T x} G_{R x} 10^{-\frac{P L}{10}},
$$

where the the $T x$ and $R x$ antenna gains are $G_{T x}$ and $G_{R x}$, respectively. $P L$ is the path loss.

In this work, since the simplified PL model (PLM) from [13], applied in simulations of dual-stripe or 5x5 Grid topologies in urban environments, did not consider the number of walls, the WINNER II path model for the sub-6 $\mathrm{GHz}$ frequency band [9] is considered. It and stands as:

$$
P L_{T x}(x, y)=A * \log _{10}(d)+B+C * \log _{10}\left(\frac{f_{c}}{5}\right)+X,
$$

where $f_{c}$ is the frequency, in $\mathrm{GHz}$, the fitting parameter $A$ includes the path loss exponent, parameter $B$ is the intercept while $C$ describes the path loss frequency dependence. One 
assumes that the UE is served by the transmitter node of that room, in Line-of-Sight (LoS). The interferer node is in Non Line-of-Sight (NLoS). For LoS scenario, $A=18.7, B=46.8$, and $C=20$; when the UE is in NLoS. Parameters take the following values: $A=20, B=46.4$ and $C=20$. $X$ is an environmentspecific term that represents the effect of wall attenuation. In the case of internal walls this attenuation is equal to $5 \mathrm{~dB}$ for each wall (or circa $10 \mathrm{~dB}$ for thick walls), meaning that $X=5 n_{W}$, where $n_{W}$ is the number of walls between the UE and transmitter or interferer. For a LoS link, the number of walls is zero and $X$ is equal to $0 \mathrm{~dB}$. In this scenario of interference, UEs are in NLoS and the number of walls is one. Hence, $X$ is equal to $5 \mathrm{~dB}$.

The average SINR is measured by an UE with uniform probability density function for its deployment over the room area. For the considered scenario the average received powers from the own node and interferer node can be expressed as follows:

$$
\begin{aligned}
\bar{P}\left(l, P_{T x}\right)= & \int_{y} \int_{x} \frac{P_{T x} G_{T x} G_{R x}}{A_{o w}} \\
& 10^{-\frac{A * \log _{10} d+B+C * \log _{10}\left(\frac{f_{c}}{5}\right)+x}{10}} d x d y,
\end{aligned}
$$

where $A_{o w}$ is the total area integration and is equal to the square of the side length of the rooms. The integration limits are equal for both integrals and are $[-l / 2, l / 2]$. The average received powers allow us to determine the average SINR.

One assumes a variation of the transmitter power of the nodes from $0 \mathrm{dBm}$ up to $20 \mathrm{dBm}$, as in [13]. Transmitter and receiver gains are assumed to be $0 \mathrm{dBm}$. The parameters for the WINNER II PLM are presented in Tab. I.

The second scenario considers three rooms deployed sideby-side, as shown in Fig. 3. When a frequency reuse equal to two is considered the interferer node is located at room 3 in the coordinates $(0,2 \times l)$. The node located at the coordinates $(0, l)$ is operating in a different carrier, with a different set of sub-channels, so the interference in the other two nodes is null.

TABLE I: WINNER II PLM parameters.

\begin{tabular}{|c|c|c|c|}
\hline \multicolumn{4}{|c|}{ PLM Parameters } \\
\hline \multicolumn{2}{|c|}{ LoS } & \multicolumn{2}{c|}{ NLoS } \\
\hline $\mathrm{A}$ & 18.7 & $\mathrm{~A}$ & 20 \\
$\mathrm{~B}$ & 46.8 & $\mathrm{~B}$ & 46.4 \\
$\mathrm{C}$ & 20 & $\mathrm{C}$ & 20 \\
$\mathrm{X}$ & $5 n_{W}$ & $\mathrm{X}$ & $5 n_{W}$ \\
$n_{W}$ & 0 & 2 rooms, $n_{W}$ & 1 \\
& & 3 rooms, $n_{W}$ & 2 \\
\hline
\end{tabular}

To determine the SINR by considering Equation 1, the coordinates of the interferer change (compared to the first scenario, presented above), and so changesthe distance in the PL equation (Equation 4). The number of walls is two, so $X$ is now equal to $10 \mathrm{~dB}$.

Results for the average SINR are presented in Fig. 4 for both scenarios. With two rooms, the average SINR is 8.65 $\mathrm{dB}$. With three rooms, due to the farthest distance between the

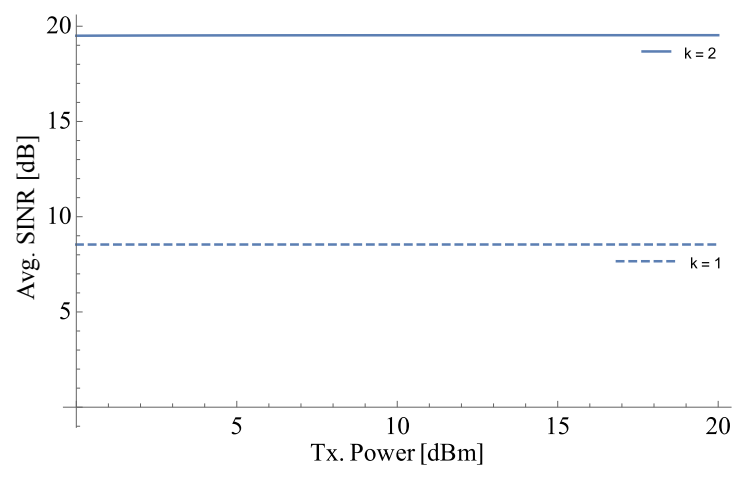

Fig. 4: Theoretical average SINR. Dashed line presents results for the 2 rooms scenario $(k=1)$, while the solid line corresponds to the 3 rooms scenario $(k=2)$.

two nodes, the average SINR increases up to $19.53 \mathrm{~dB}$. It is also worthwhile to mention that, as the system is interference limited, the variation of the transmitter power has not produced a noticeable impact on the average SINR.

\section{Exponential Effective SINR Mapping Study}

EESM is a channel-dependent formulation that is represented by an equation which maps the power level and MCS to the values generated by each SINR in the domain [14]. To simulate the EESM on the room of interest, an upgraded version of the LTE-Sim was used [11], [15]. A transmitter power of $20 \mathrm{dBm}$ was considered together with receiver and transmitter gains of $0 \mathrm{dBi}$. Tab. II presents the parameters considered to perform the simulations.

TABLE II: Simulation parameters to obtain the EESM.

\begin{tabular}{|c|c|}
\hline \multicolumn{2}{|c|}{ LTE-Sim parameters } \\
\hline Packet scheduler & Proportional fair \\
CQI report & Periodic \\
CQI report interval & $1 \mathrm{~ms}$ \\
Frequency reuse pattern & $1 ; 2$ \\
Noise figure & 2.5 \\
\hline
\end{tabular}

Fig. 5 presents the simulation results for EESM. In the topology with two contiguous rooms, the lowest values of EESM are obtained if UEs are near the wall shared with the neighbor room, as shown in Fig. 5a. In this case, the EESM is $\approx 5 \mathrm{~dB}$; in the opposite wall the ESSM is $\approx 11.5 \mathrm{~dB}$. The highest values are obtained near the eNodeB.

This behavior is maintained when 3 rooms are considered, and the interference comes from room 3 only. Results are shown in Fig. 5b. EESM is higher, as the interferer node is farther away. The lowest obtained value of EESM is $17 \mathrm{~dB}$, near the wall that separates room 1 from room 2, while in the opposite wall the value of ESSM is $21 \mathrm{~dB}$. The maximum possible value of $40 \mathrm{~dB}$ for EESM was obtained in a broad area near the cell center. 


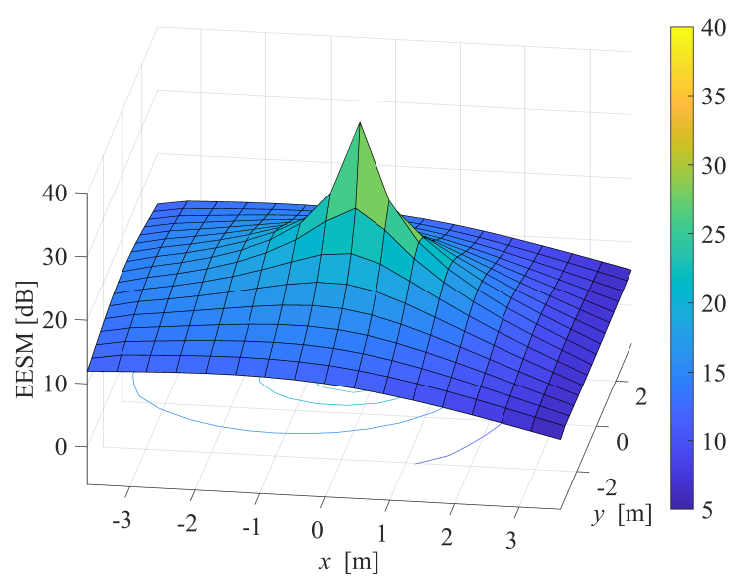

(a) room $2(k=1)$

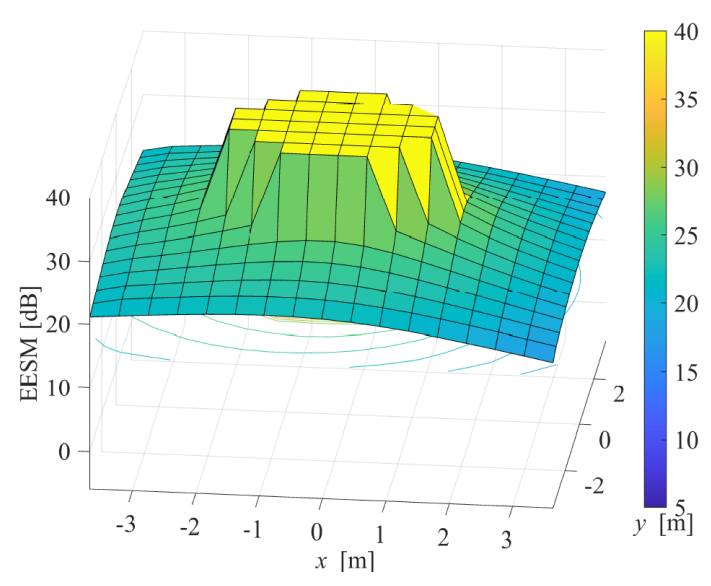

(b) room $3(k=2)$

Fig. 5: EESM for a frequency band of $2.6 \mathrm{GHz}$ with $10 \mathrm{MHz}$ frequency bandwidth.

\section{Received Power Measurements SRsLTE System}

\section{A. Environment Characterization and Experimental Setup}

The experimental setup features one or two base stations that are supported by USRP B210 together with srsLTE running over Linux Mint v20.1 in portable computers [16]. These computers support the eNodeBs and evolved packet core (EPC). Similarly, authors from [17] used USRPs B200mini and B205-mini to establish a testbed setup for LTE. In a slightly different way, authors from [18] considered USRPs B210 to show the interoperability between LTE and WiFi to offload the $4 \mathrm{G}$ mobile communications traffic to the WLAN.

SrsLTE [19], [20] is executed over GNU Radio [21] to make use of resources such as the built-in spectrum analyzer. The library supports different types of radio frequency (RF) frontends [22]. The supported bandwidths are the 1.4, 3, 5, 10 and $20 \mathrm{MHz}$ [4]. The srsLTE maps the signal-to-noise ratio (SNR) performance into BLER in real time, during the tests.

Rooms are characterized by the ceiling made by mineral fiber boards (whose height is $3 \mathrm{~m}$ ), glass windows, and projected plaster walls. The main base station (BS), supported by USRP B210, was at room 1, while the interferer BS may be at room 2 or room 3 . BSs were fixed close to the ceiling, at the center of the rooms. The UE was positioned at $1.5 \mathrm{~m}$ height, as shown in Fig. 6.

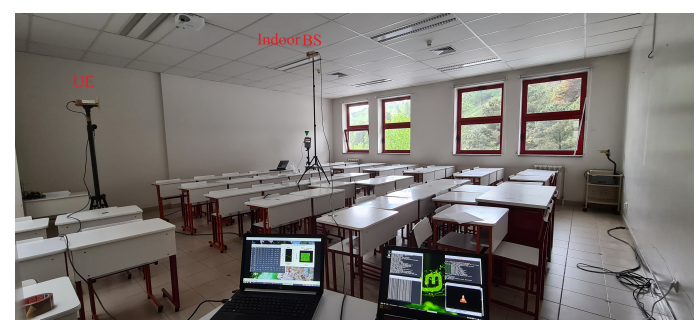

Fig. 6: Experimental setup with USRP B210 and PCs running srsLTE.

USRP B210 supports a sampling rate of $61.44 \mathrm{MHz}$, continuous frequency ranging from $70 \mathrm{MHz}$ up to $6 \mathrm{GHz}$ and enables flexible $R x$ gain adjustment [10]. Each USRP is connected to a notebook (equipped with USB 3.0 and Intel $^{\odot}$ Core $^{\mathrm{TM}}$ i7$8770 \mathrm{CPU}$ with four physical cores with a maximum clock frequency of $4.0 \mathrm{GHz}$ ) running the srsLTE, as shown in Fig. 6. All the notebooks have a similar configuration.

\section{B. Testing with Software defined radio}

4G networks provide high-quality access to mobile users while supporting enough user throughput, even for users further away distant from $T x$ nodes. As frequency channels need to be reused in different zones of the buildings, the impact of the interference between co-channel cells needs to be assessed through the evaluation of $C / I$.

By considering video streaming transmission, the study of srsLTE system performance mainly involves to measure the $C / I$ for the DL, as well as the throughput and delay of the system. The srsLTE execution parameters are configured through the configuration file (.config), i.e., only the configuration file is added after the program is running and the configuration parameters are executed in the configuration file. The change in the output power of the USRP with the srsLTE is made through the (.config/srsenb.config) file, where it is possible to configure the parameters, e.g, frequency or gains. The srsLTE identifies only frequencies in E-UTRA absolute radio-frequency channel number (EARFCN), in addition to varying the frequency. Tests with srsLTE have been carried out with EARFCN 2800, corresponding to the $2625 \mathrm{MHz}$ frequency band (10 MHz bandwidth).

\section{Results and Analysis}

Tests have been performed with video streaming (at 3.1 $\mathrm{Mb} / \mathrm{s}$ ) running in the transmitter mode and being received in the UE. Measurements have been made by collecting data during sixty seconds in each of the 49 points.

First, we have extracted results for the received power in the 49 measurement points of the topology without any interferer, i.e., with only one transmitter node in room 1 . The position of the UE varies inside the room in the considered $(x, y)$ 
coordinates. Fig. 7 shows the average received power as a function of the position within the classroom in a scenario without interference nodes.

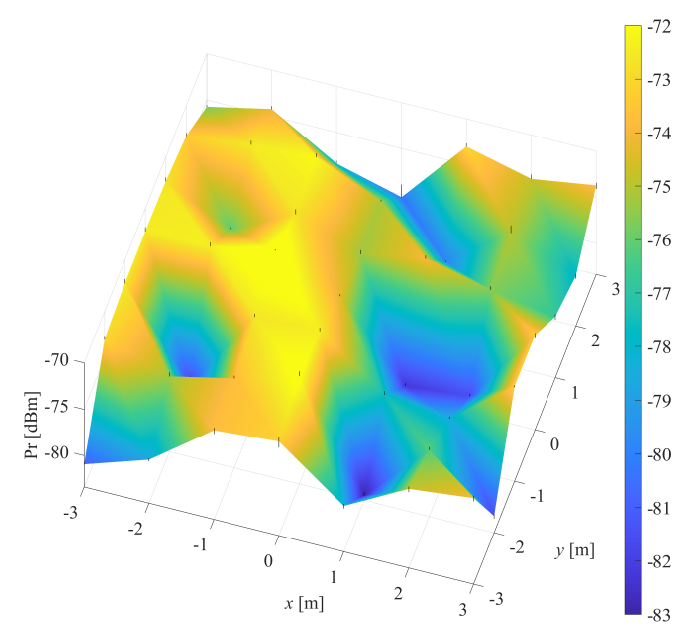

Fig. 7: Received Power for the $2.6 \mathrm{GHz}$ frequency band, measured with USRP B210, without interferer.

In the presence of interference, as a trend, there is a reduction in $C / I$ when the $\mathrm{UE}$ walks towards the interferer. Besides, a difference of more than $10 \mathrm{~dB}$ exists in the results for the average $C / I$ with the interferer BS placed in room 2 (Fig. 9a) and in room 3 (Fig. 9b), partly explained $(\approx 9 \mathrm{~dB})$ explained by the attenuation in each wall.

Fig. 10 compares the values of $C / I$, with received powers from the own cell and interferer nodes) measured with the USRPs B210 (and srsLTE) with analytical results obtained with the Winner II path loss model (curves in dark or light blue if interference comes from rooms 2 or 3, respectively), over the straight line in the centre of the rooms $(y=0)$, common to all the BSs (represented by dark orange dots or light orange squares, if interference comes from rooms 2 or 3 , respectively). Measurement results with an FSH8 spectrum analyzer are also presented (with dark purple dots diamonds or light purple triangles if interference comes from rooms 2 or 3 , respectively). As measurements have been taken simultaneously, differences between FSH 8 and USRPs measurements were caused by an hiding effect of a device to the other.

\section{CONCLUSION}

We have considered 4G indoor coverage on a floor of our Department with adjacent classrooms. The size of each room is $7.32 \times 7.32 \mathrm{~m}^{2}$. While room 1 is the room of interest, where theoretical and practical measurements took place, BSs that act as wireless interfering nodes are also separately considered either in room 2 or room 3 . By varying the UE positions within room 1, it was possible to verify that the highest values of the received power occur close to the central BS. However, compared to the WINNER II propagation model, the received power does not decrease suddenly because of the effect of the reduced gain in the radiation pattern in the back part of the antenna. As the system is interference limited, as a general trend, results for $C / I$ follow the behavior of the EESM curves. $C / I$ is higher in the wall further away from the interferer, and decreases when the UEs comes close by the wall that divides rooms 1 and 2 . In addition, it was demonstrated that there is an effect of "wall loss" shown by the path loss increase between room 2 and room 1 (or between room 2 and 3 ). If we consider an attenuation for each wall of circa 7-9 $\mathrm{dB}$ the trend of the WINNER II model for the interference coming across different walls is verified. Future work includes to analyze in detail results obtained with the spectrum analyzer while considering upper frequency bands that are being considered for $5 \mathrm{G} \mathrm{New}$ Radio and will allow for supporting larger bandwidths, e.g., at the $3.5 \mathrm{GHz}$ and $5.4 \mathrm{GHz}$ frequency bands.A vector network generator may also be used.

\section{REFERENCES}

[1] 3GPP, “TR 21.916 Release 16 Description; Summary of Rel-16 Work Items," 3rd Generation Partnership Project, Tech. Rep. V1.0.0, Dec. 2020. [Online]. Available: https://www.3gpp.org/ftp/Specs/archive/21_sseries/21.916/21916100.zip

[2] J. Mitola, "Software radios-survey, critical evaluation and future directions," in [Proceedings] NTC-92: National Telesystems Conference, May 1992, pp. 13/15-13/23.

[3] W. H. Tuttlebee, Software defined radio: enabling technologies. John Wiley \& Sons, 2003.

[4] I. Gomez-Miguelez, A. Garcia-Saavedra, P. D. Sutton, P. Serrano, C. Cano, and D. J. Leith, "SrsLTE: An Open-Source Platform for LTE Evolution and Experimentation," in Proceedings of the Tenth ACM International Workshop on Wireless Network Testbeds, Experimental Evaluation, and Characterization, ser. WiNTECH '16. New York, NY, USA: Association for Computing Machinery, 2016, p. 25-32. [Online]. Available: https://doi.org/10.1145/2980159.2980163

[5] A. Puschmann, P. Sutton, and I. Gomez, "Implementing NB-IoT in Software - Experiences Using the srsLTE Library," 2017.

[6] S. Jain, Y. Zhang, and L. A. DaSilva, "LTE Standard-Compliant D2D Communication: Software-defined Radio Implementation and Evaluation," CoRR, vol. abs/1904.12934, 2019. [Online]. Available: http://arxiv.org/abs/1904.12934

[7] I. Gomez-Miguelez, A. Garcia-Saavedra, P. D. Sutton, P. Serrano, C. Cano, and D. J. Leith, "SrsLTE: An Open-Source Platform for LTE Evolution and Experimentation," in Proceedings of the Tenth ACM International Workshop on Wireless Network Testbeds, Experimental Evaluation, and Characterization, ser. WiNTECH '16. New York, NY, USA: Association for Computing Machinery, 2016, p. 25-32. [Online]. Available: https://doi.org/10.1145/2980159.2980163

[8] D. M. Rose, T. Jansen, and T. Kürner, "Indoor to outdoor propagation - Measuring and modeling of femto cells in LTE networks at 800 and $2600 \mathrm{MHz}$," in 2011 IEEE GLOBECOM Workshops (GC Wkshps), Dec 2011, pp. 203-207.

[9] P. Kyösti, J. Meinilä, L. Hentilä, X. Zhao, T. Jämsä, C. Schneider, M. Narandzić, M. Milojević, A. Hong, J. Ylitalo, V. Holappa, M. Alatossava, R. Bultitude, Y. de Jong, and T. Rautiainen, "IST-4-027756 WINNER II D1.1.2 V1.2 WINNER II Channel Models," Information Society Technologies, Germany, Tech. Rep. D1.1.2 V1.2, February 2008. [Online]. Available: http://www.cept.org/files/1050/documents/winner2 - final report.pdf

[10] ettus, "USRP B200/B210 Bus Series Specifications," http://www.ettus.com/content/files/b200-b210_spec_sheet.pdf, accessed: 2020-23-03.

[11] R. R. Paulo and F. J. Velez, "An Extensive Study on the Performance Evaluation and Scheduling of HeNBs," IEEE Access, vol. 9, pp. 40098 $40110,2021$.

[12] K. Chen and J. de Marca, Mobile WiMAX, ser. Wiley IEEE. John Wiley \& Sons, Ltd, 2008. [Online]. Available: https://books.google.es/books?id=7TWzvbZkLMgC 


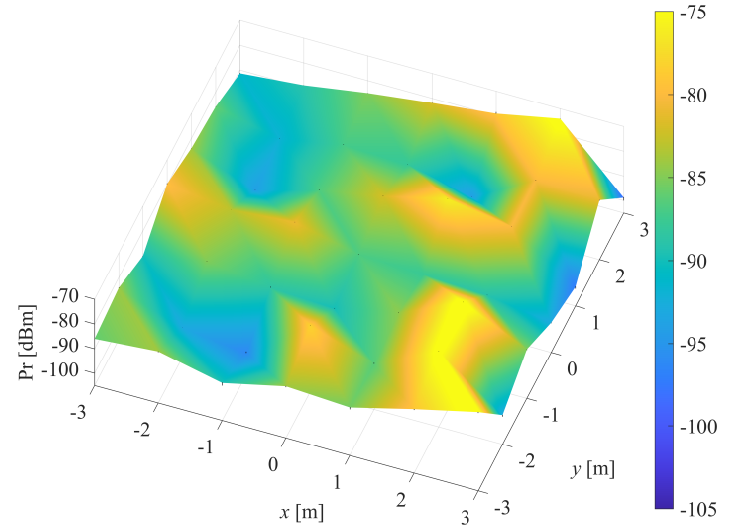

(a) Interferer in room $2(k=1)$

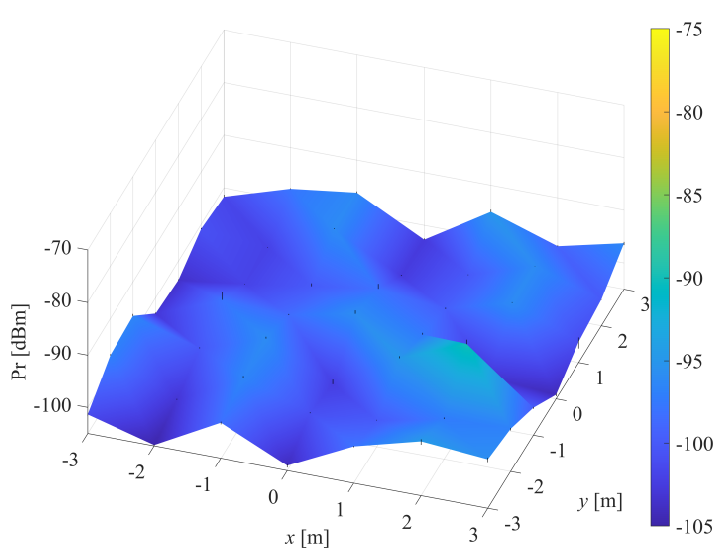

(b) Interferer in room $3(k=2)$

Fig. 8: $P_{r}$ from the interference nodes located in room 2 or 3 measured with USRP B210, for $f=2.6 \mathrm{GHz}$.

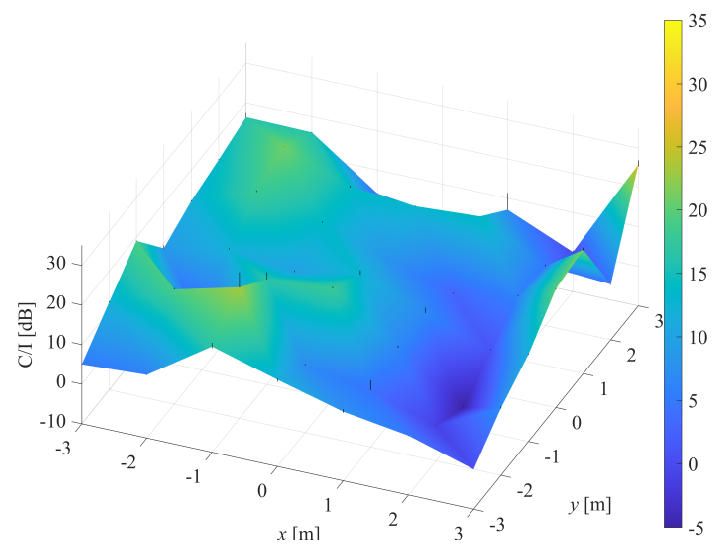

(a) Interferer in room $2(k=1)$

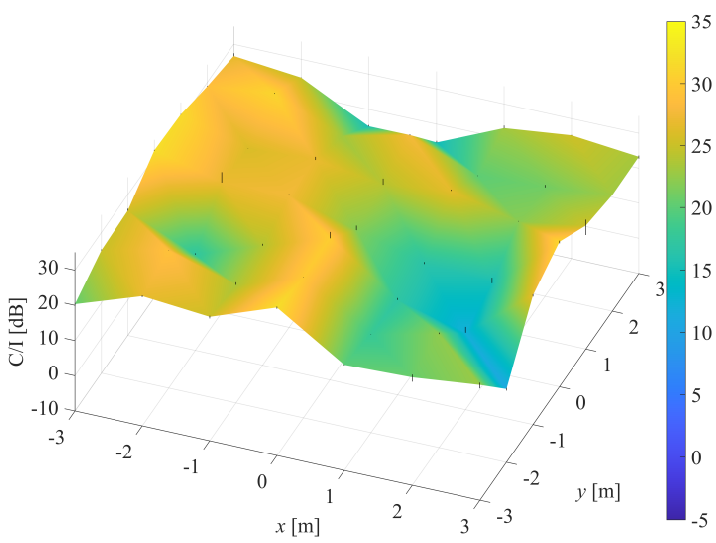

(b) Interferer in room $3(k=2)$

Fig. 9: $C / I$ in room1, with interference coming either from rooms 2 or 3 , measured with USRP B210, for $f=2.6 \mathrm{GHz}$.

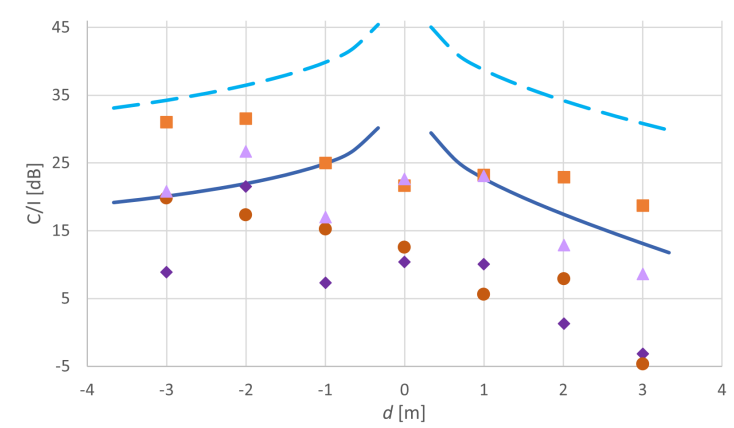

Fig. 10: Comparison of the $C / I$ resulting from the Winner II model (in blue), or from measurements with USRPs (orange) or spectrum analyzer (purple) - N.B.: dark or light colours correspond to interference from rooms 1 and 2 , respectively.

[13] 3GPP, "R4-092042 Simulation assumptions and parameters for FDD HeNB RF requirements," 3rd Generation Partnership Project, Tech. Rep. Meeting \#51, May 2009. [Online]. Available: https://www.3gpp.org/ftp7tsg_ran/WG4_Radio/TSGR4_51/Documents/ R4_092042.zip

[14] S. Mumtaz and A. Gameiro, "Enhanced Algorithm for Link to System
Interface Mapping," in (IJCSIS) International Journal of Computer Science and Information Security, vol. 3, no. 3, Jul. 2009, pp. 1-3.

[15] G. Piro, L. A. Grieco, G. Boggia, F. Capozzi, and P. Camarda, "Simulating LTE Cellular Systems: An Open-Source Framework," IEEE Trans. on Vehicular Technology, vol. 60, no. 2, pp. 498-513, Feb 2011.

[16] L. M. Team. (2021) Linux Mint. [Online]. Available: https://linuxmint.com/download.php

[17] A. Hematian, J. Nguyen, C. Lu, W. Yu, and D. Ku, "Software defined radio testbed setup and experimentation," in Proceedings of the International Conference on Research in Adaptive and Convergent Systems, ser. RACS '17. New York, NY, USA: Association for Computing Machinery, 2017, p. 172-177. [Online]. Available: https://doi.org/10.1145/3129676.3129690

[18] K. Abbas, M. Afaq, T. Ahmed Khan, A. Rafiq, J. Iqbal, I. U1 Islam, and W.-C. Song, "An efficient sdn-based lte-wifi spectrum aggregation system for heterogeneous $5 \mathrm{~g}$ networks," Transactions on Emerging Telecommunications Technologies, vol. n/a, no. n/a, p. e3943. [Online]. Available: https://onlinelibrary.wiley.com/doi/abs/10.1002/ett.3943

[19] srsLTE. (2020) Software Radio Systems - srsLTE 20.10.1 Documentation. [Online]. Available: https://docs.srslte.com/en/latest/

[20] srsLTE_git. (2020) Software Radio Systems "srsLTE: Open Source LTE. [Online]. Available: https://github.com/srsLTE/srsLTE

[21] G. R. project. (2021) GNU Radio. [Online]. Available: https://www.gnuradio.org/

[22] N. Nikaein, "OpenAirInterface Simulator/Emulator," 2015. 